\title{
ACCUMULATED DAMAGE EVOLUTION AND INVESTMENT COSTS OF BREAKWATERS
}

$\underline{\text { P.Folgueras }}^{(1)}$, folgueras@ugr.es, J. Del-Rosal-Salido(1), jrsalido@ugr.es, M. V.Moragues ${ }^{(1)}$, moraguesgomez@gmail.com J.D. Lopez ${ }^{(2)}$, jdamian@puertos.es, M.A. Losada(1), mlosada@ugr.es

(1) IISTA, Universidad de Granada, (2) Puertos del Estado, M. de Fomento - España

\begin{abstract}
JUSTIFICATION, MOTIVATION AND OBJECTIVE
Since the pioneering work by Borgman, (for vertical walls), and Paape, (for rubble mound breakwaters) in the 50's of the last century, the design of new breakwaters and rehabilitation of existing breakwaters can be based on lifecycle analysis targeting the optimum investment costs, including the construction costs, maintenance, repairs and demolition, costs, and downtime costs. The estimation of these costs is based on the performance criteria of each failure mode for the structural and hydraulic responses related to the design limit stages SLS and ULS, including both a physical measure and a probability of occurrence, ROM 0.0 and 1.0 (2000 and 2007) and PIANC 2017. To evaluate these costs including their uncertainty it is necessary to apply, (1) a spatial and temporal damage evolution model of each failure mode, (2) to define the interdependence between modes including the decision criteria when start to repair, and (3) to compute the costs depending on the real sea conditions, the strategy of construction and the available construction power.
\end{abstract}

The main objective of this work is to present a methodology to estimate the probability distribution function of the investment costs in the useful life of the structure

\section{METHODOLOGY}

The flow chart of the present method includes the selection of the typology for each of the stretches defining the plan of the breakwater, and for each stretch, the diagrams of failure and a model of evolution of failure. Next, the definition, (during the design process), of the interdependence and triggering of failure modes, and the development of a strategy of construction and reparation summarized by the corresponding decision trees. Finally, the method includes a simulation algorithm of the climatic agents and a module to simulate the construction processes and to compute the actual costs of the construction and the reparation in the useful life of the structure. The evolution of the damage is based on the stochastic model for damage accumulation proposed by Castillo et al. 2012. The simulation process is based on a non-stationary parametric distribution to model the multivariate distribution of the main maritime variables, a vector autoregressive (VAR) model to describe their temporal dependence, and the use of copulas to link the temporal dependency of the storm duration and the inter-arrival time between two consecutive storms.

\section{RESULTS AND CONCLUSIONS}

The figure shows the typology, the accumulated damage of the failure mode FM1 (stability of armor units) due to the storm defined as histograms of sea states, a repair strategy and the output of the model including the accumulated damage of the failure modes FM1, FM2 and $F M 3$, the repairs and the temporal evolution of the costs. The pdf of the investment costs depends on the pdf of the climate agents, the design process and the adopted strategies during the construction and the reparations.
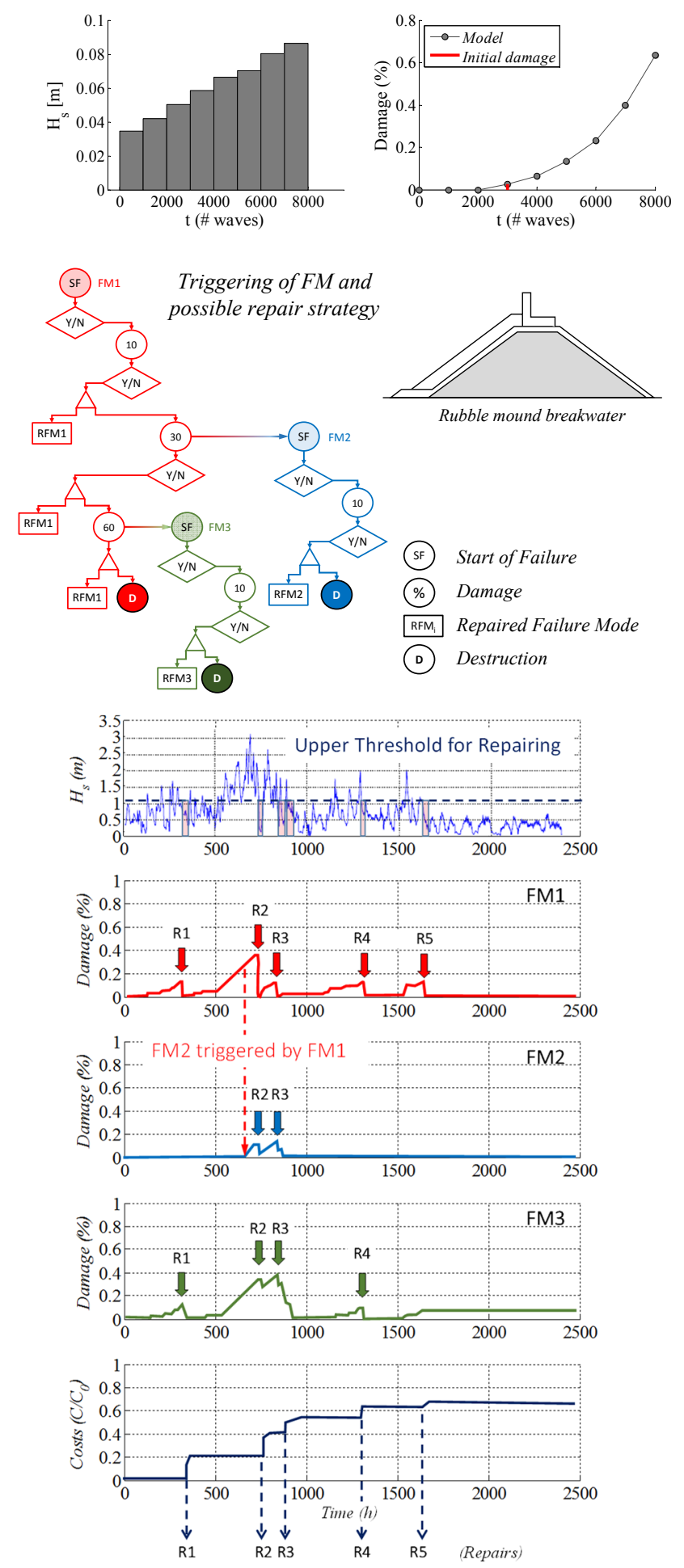

Figure 1. Some preliminary results

\section{REFERENCES}

Castillo et al., 2012, J. of WPCO, ASCE Vol. 138, No. 6 PIANC, 2016, Report no 196, pp.179

ROM 0.0-1.0, 2000-2007 Puertos del Estado. Madrid 